\title{
The effects of spatial and temporal replicate sampling on eDNA metabarcoding
}

\author{
Kevin K Beentjes ${ }^{\text {Corresp., } 1,2}{ }^{\text {, Arjen GCL Speksnijder }}{ }^{1}$, Menno Schilthuizen ${ }^{1,2}$, Marten Hoogeveen ${ }^{1}$, Berry B van der \\ Hoorn ${ }^{1}$ \\ 1 Naturalis Biodiversity Center, Leiden, The Netherlands \\ 2 Institute of Biology Leiden, Leiden University, Leiden, The Netherlands \\ Corresponding Author: Kevin K Beentjes \\ Email address: Kevin.Beentjes@naturalis.nl
}

Background. The heterogeneous nature of environmental DNA (eDNA) and its effects on species detection and community composition estimates has been highlighted in several studies in the past decades. Mostly in the context of spatial distribution over large areas, in fewer occasions looking at spatial distribution within a single body of water. Temporal variation of eDNA, similarly, has mostly been studied as seasonality, observing changes over large periods of time, and often only for small groups of organisms such as fish and amphibians.

Methods. We analyzed and compared small-scale spatial and temporal variation by sampling eDNA from two small, isolated dune lakes for twenty consecutive weeks. Metabarcoding was performed on the samples using generic COI primers. MOTUs were used to assess dissimilarities between spatial and temporal replicates.

Results. Our results show large difference between samples taken within one lake at one point in time, but also expose the large differences between temporal replicates, even those taken only one week apart. Furthermore, between-site dissimilarities showed a linear correlation with time frame, indicating that between-site differences will be inflated when samples are taken over a period of time. We also assessed the effects of PCR replicates and processing strategies on general patterns of dissimilarity between samples. While more inclusive PCR replicate strategies lead to higher richness estimations, dissimilarity patterns between samples did not significantly change.

Conclusions. We conclude that the dissimilarity of temporal replicates at a one week interval is comparable to that of spatial replicate samples. It increases, however, for larger time intervals, which suggests that population turnover effects can be stronger than community heterogeneity. Spatial replicates alone may not be enough for optimal recovery of taxonomic diversity, and cross-comparisons of different locations are susceptible to inflated dissimilarities when performed over larger time intervals. Many of the observed MOTUs could be classified as either phyto- or zooplankton, two groups that have gained traction in recent years as potential novel bio-indicator species. Our results, however, indicate that these groups might be susceptible to large community shifts in relatively short periods of time, highlighting the need to take temporal variations into consideration when assessing the usability as water quality indicators. 


\section{The effects of spatial and temporal replicate sampling on eDNA metabarcoding}

2

3 Kevin K. Beentjes ${ }^{1,2}$, Arjen G. C. L. Speksnijder ${ }^{1}$, Menno Schilthuizen ${ }^{1,2}$, Marten Hoogeveen ${ }^{1}$,

4 Berry B. van der Hoorn ${ }^{1}$

5

$6 \quad{ }^{1}$ Naturalis Biodiversity Center, Leiden, the Netherlands

$7 \quad{ }^{2}$ Institute of Biology Leiden, Leiden University, the Netherlands

9 Corresponding author:

Kevin K. Beentjes

Naturalis Biodiversity Center, PO box 9517, 2300 RA Leiden, the Netherlands

Email address: kevin.beentjes@naturalis.nl

\section{ABSTRACT}

Background. The heterogeneous nature of environmental DNA (eDNA) and its effects on species

detection and community composition estimates has been highlighted in several studies in the past

decades. Mostly in the context of spatial distribution over large areas, in fewer occasions looking at spatial distribution within a single body of water. Temporal variation of eDNA, similarly, has mostly been studied as seasonality, observing changes over large periods of time, and often only for small groups of organisms such as fish and amphibians. Methods. We analyzed and compared small-scale spatial and temporal variation by sampling eDNA from two small, isolated dune lakes for twenty consecutive weeks. 
23 Metabarcoding was performed on the samples using generic COI primers. MOTUs were used to assess dissimilarities between spatial and temporal replicates.

Results. Our results show large difference between samples taken within one lake at one point in time, but also expose the large differences between temporal replicates, even those taken only one week apart. Furthermore, between-site dissimilarities showed a linear correlation with time frame, indicating that between-site differences will be inflated when samples are taken over a period of time. We also assessed the effects of PCR replicates and processing strategies on general patterns of dissimilarity between samples. While more inclusive PCR replicate strategies lead to higher richness estimations, dissimilarity patterns between samples did not significantly change.

Conclusions. We conclude that the dissimilarity of temporal replicates at a one week interval is comparable to that of spatial replicate samples. It increases, however, for larger time intervals, which suggests that population turnover effects can be stronger than community heterogeneity. Spatial replicates alone may not be enough for optimal recovery of taxonomic diversity, and cross-comparisons of different locations are susceptible to inflated dissimilarities when performed over larger time intervals. Many of the observed MOTUs could be classified as either phyto- or zooplankton, two groups that have gained traction in recent years as potential novel bio-indicator species. Our results, however, indicate that these groups might be susceptible to large community shifts in relatively short periods of time, highlighting the need to take temporal variations into consideration when assessing the usability as water quality indicators.

\section{INTRODUCTION}

The importance of freshwater biodiversity and its effects on ecosystem resilience and stability have been well documented, and its monitoring is regulated by legislation such as the European Union Water 
47 Framework Directive of 2000 (EU WFD; Directive 2000/60/EC). Monitoring of biological quality elements

48 (BQE), such as macroinvertebrates, is prescribed under the WFD, but traditional methods employed in

49 this field are often considered slow, expensive, and sensitive to human-induced bias and errors (e.g.

Clarke \& Hering 2006). Integration of molecular tools has been a focal area within this field of research

for the past decade. The use of environmental DNA (eDNA) metabarcoding for species detection is

gaining traction, as it would potentially enable to circumvent cumbersome traditional collection or visual

observation of specimens. The use of eDNA for detection is based on the fact that organisms living in a

certain environment, such as freshwater, leave behind traces of their existence via shedding and

excretion of DNA. This technique has been applied successfully for the detection of a multitude of

and invertebrates (Thomsen et al., 2012; Schneider et al., 2016; Klymus, Marshall \& Stepien, 2017).

The heterogeneous nature of eDNA has been investigated in several model organisms, for example amphibians, where it was shown that spatial sampling increased the detection probability (Dejean et al., 2012; Schmidt et al., 2013). Similarly, richness estimates from eDNA community metabarcoding are sensitive to sampling strategies (Grey et al., 2018). This suggests that eDNA may only represent very local signals, especially in standing waters. It is therefore often recommended to include spatial coverage in an eDNA sampling strategy, either by sampling various points within a water body, or by combining all these samples into one large sample representing the entire water body (Goldberg et al., 2016; Grey et al., 2018; Harper et al., 2019). In addition to spatial sampling, temporal replicates may also increase detection probability, and provide a more complete impression of species richness and community composition. Many studies have examined the effects of spatial and temporal sampling on (macroinvertebrate) communities (e.g. Baselga et al. 2013, Barsoum et al. 2019), but limited work has 
71 groups of organisms, such as fish (Stoeckle, Soboleva \& Charlop-Powers, 2017; Sigsgaard et al., 2017),

72 amphibians (Rees et al., 2017; Buxton, Groombridge \& Griffiths, 2018), and chironomids (Bista et al.,

73 2017), or assesses the seasonal differences only at a limited number of points in time (Chain et al., 2016;

74 Guardiola et al., 2016).

75

In this paper we compare the effects of both spatial and temporal replicate sampling of eDNA within

two isolated, but nearby, lakes, using a generic COI primer set. We assess patterns in communities based on MOTU clustering, identifying MOTUs using a lowest common ancestor (LCA) approach, and also look at the communities of only those MOTUs identified as metazoans. Furthermore, we assess the impact of PCR replicates and subsequent sequence or bioinformatics processing strategies on the observed patterns of eDNA through space and time. We also highlight some potential opportunities and caveats in the use of eDNA for freshwater quality monitoring.

\section{MATERIALS \& METHODS}

\section{Field Sampling}

87 Samples were collected on every Monday for 20 consecutive weeks, from May 2016 to September 2016,

88 from two permanent lakes in a Natura 2000 protection area in the dunes of Wassenaar, the Netherlands. Two locations were selected, approximately 1.9 kilometers apart: Location 1 "De

91 window coincides with the sampling period for traditional WFD monitoring. Within each location three

92 sub-sites were selected around the lake, roughly equidistant from each other (40-60 meters apart) and representing different habitats and substrates. One liter of water was taken by submerging a one-liter

94 sterile bottle slightly below the surface, one meter away from the lake shoreline. The bottles were 
95

97

brought back to the laboratory for filtration. As the sites were located in a nature conservation area, a permit was obtained from Staatsbosbeheer (2016/022).

\section{DNA filtration and extraction}

Environmental DNA (eDNA) filtration was performed in the laboratory within 4 hours after collecting the samples in the field. Sterilized Nalgene filter units (Thermo Fisher, Waltham, MA, USA) attached to a vacuum pump with $0.2 \mu \mathrm{m}$ polyethersulfone (PES) filter membranes (Sartorius, Göttingen, Germany) were used to filter $300 \mathrm{ml}$ of water. Filter holders were sterilized using $10 \%$ bleach solution and placed under UV-light for 30 minutes before use. After filtration, the filter membranes were stored in $900 \mu l$

CTAB buffer at $-20^{\circ} \mathrm{C}$ until extraction. DNA was extracted using a modified CTAB extraction protocol, adapted from Turner et al. (2014). DNA precipitation was performed on $800 \mu$ l of aqueous phase, and final resuspension of the pellet was performed in $50 \mu \mathrm{l}$ AE buffer (Qiagen, Venlo, the Netherlands).

\section{DNA amplification and MiSeq sequencing}

A 316 bp fragment of the COI barcode region was amplified using primers BF1 and BR2 (Elbrecht \& Leese, 2017). All sampling replicates were amplified in three independent PCRs, which were sequenced separately without pooling. A dual indexed MiSeq amplicon library was prepared using a two-step PCR protocol, in which the first PCR used primers BF1 and BR2 with $5^{\prime}$ Illumina tails (Tables S1-S2). PCRs for round 1 were performed in $25 \mu$ reactions containing 1x Qiagen CoralLoad PCR Buffer, 0.5 mM dNTPs, $0.05 \mathrm{U} / \mu \mathrm{l}$ Taq polymerase (Qiagen, Venlo, the Netherlands), $0.4 \mu \mathrm{M}$ of each primer and $1.0 \mu \mathrm{l}$ of template DNA. Initial denaturation was performed at $94^{\circ} \mathrm{C}$ for 3 minutes, followed by 40 cycles at $94^{\circ} \mathrm{C}$ for 15 seconds, $50^{\circ} \mathrm{C}$ for 30 seconds and $72^{\circ} \mathrm{C}$ for 40 seconds, followed by final elongation at $72^{\circ} \mathrm{C}$ for 5 minutes. Each 96-well plate contained blanks with no template DNA and positive controls of Reeve's muntjac (Muntiacus reevesi) DNA extract to enable detection of cross-contaminations in the laboratory 
119 process. PCR success was checked on an E-Gel 96 pre-cast agarose gel (Thermo Fisher, Waltham, MA, 120 USA). PCR products where then cleaned with a one-sided size selection using NucleoMag NGS-Beads 121 (Macherey-Nagel, Düren, Germany), a 1:0.9 ratio.

122

123 Second round PCRs were performed using $2.0 \mu \mathrm{l}$ of PCR product from the first round in a $20 \mu \mathrm{l}$ reaction 124 containing 1x TaqMan Environmental Master Mix 2.0 (Thermo Fisher, Waltham, MA, USA) and $1.0 \mu \mathrm{M}$ of 125 each primer. Initial denaturation was performed at $95^{\circ} \mathrm{C}$ for 10 minutes, followed by 11 cycles at $95^{\circ} \mathrm{C}$ 126 for 30 seconds, $55^{\circ} \mathrm{C}$ for 60 seconds and $72^{\circ} \mathrm{C}$ for 30 seconds, followed by final elongation at $72^{\circ} \mathrm{C}$ for 7 127 minutes. Second round PCR products were quantified on the QIAxcel (Qiagen, Venlo, the Netherlands) and pooled equimolarly per PCR plate. Pools were cleaned with a one-sided size selection using NucleoMag NGS-Beads, ratio 1:0.9, then quantified on the Bioanalyzer 2100 (Agilent Technologies, Santa Clara, CA, USA) with the DNA High Sensitivity Kit. The four pools were combined equimolarly and sequenced on one run of Illumina MiSeq (v3 Kit, 2×300 paired-end) at LGTC (Leiden, the Netherlands).

\section{Quality filtering and MOTU clustering}

Quality filtering and clustering of all data was performed in a custom pipeline on the OpenStack environment of Naturalis Biodiversity Center through a Galaxy instance (Afgan et al., 2018). Raw sequences were filtered using Sickle (Joshi \& Fass, 2011) and merged using FLASH v1.2.11 (Magoč \& Salzberg, 2011); all non-merged reads were discarded. Samples were split based on the presence of template-specific additional bases between Illumina tail and template-specific primers with a custom tool, and primers were trimmed from both ends of the merged reads using Cutadapt v1.16 (Martin, 2011). Any read without both primers present and anchored was removed. PRINSEQ v0.20.4 (Schmieder 
143 Operational Taxonomic Unit (MOTUs) using UNOISE3 (Edgar, 2016) with an alpha of 0.5. The presence of

144 Muntiacus reevesi reads in the non-control samples was used to determine the MOTU filtering

145 threshold, only MOTUs with read abundances above $0.05 \%$ were retained for each replicate. Geneious

1468.1 .8 (https://www.geneious.com) was used to check for and remove MOTUs with indels and/or stop

147 codons.

148

149

Taxonomic assignment and diversity analysis

150 BLAST+ (Camacho et al., 2009) was used to compare MOTU sequences to a custom-made reference

151 library containing $\mathrm{COI}$ sequences and bacterial genomes downloaded from NCBI GenBank (Benson et al.,

152 2005) (sequences downloaded 21 August 2018). MEGAN v6.12.5 (Huson et al., 2007) was used to assign

153 higher-rank taxonomy to MOTUs using the lowest common ancestor (LCA) approach from the top 100

154 hits from BLAST (settings: minimum bit score 170, minimum percent identity 80 , top percent 5). The

155 VEGAN package (Oksanen et al., 2007) in R was used to calculate beta diversity (Sørenson dissimilarity)

156 between replicates and time points, make NMDS plots, and calculate correlations between dissimilarity

157 matrices and between the sample dissimilarity and sampling intervals. PCR replication effects were

158 assessed using three methods of replicate processing: (1) counting all MOTUs towards the sample

159 ("additive"), (2) only counting those MOTUs that appear in a majority of the samples ("relaxed"), or (3)

160 only counting those MOTUs that occur in all replicates ("strict") (Alberdi et al., 2018). All analysis on the

161 data were performed for both the whole dataset (all MOTUs), and a subset of the data with only

162 metazoan MOTUs.

163

164 RESULTS

165

166 Sequencing run statistics

Peer] reviewing PDF | (2019:04:36312:1:1:NEW 15 May 2019) 
167 A total of 7,692,379 read pairs were obtained after sequencing. After merging and quality filtering,

$1685,743,638$ sequences were retained for MOTU clustering. Muntiacus reevesi reads were detected in 169 several non-control samples. Using a $0.05 \%$ threshold for filtering low-abundance MOTUs from each

170 sample removed muntjac reads from all but one sample (Location 1.2, May 16). After filtering the MOTU

171 table, 1,333 MOTUs were retained in the non-control samples. An additional 19 MOTUs with indels and

172 stop codons were removed, resulting in a dataset with 1,314 MOTUs, representing 4,197,403 reads.

173 Four samples with fewer than 2,000 reads were discarded. On average, PCR replicates had 11,790 reads

174 (range 2,296-73,477), and 72 MOTUs (range 12-177). There was no correlation between number of 175 reads and number of MOTUs in each sample.

177 Taxonomic composition

178 Out of 1,314 remaining MOTUs, 530 (40.3\%) eukaryotes could be identified to at least phylum level 179 using the LCA, 119 (9.1\%) were only classified as "eukaryote", 62 (4.7\%) were identified as bacteria and

603 (45.9\%) were not assigned any classification (Fig. 1). Within the eukaryotes, most MOTUs (318) were classified as stramenopiles. Of the 176 metazoans, 121 were identified as arthropods, mostly assigned to branchiopods (44 MOTUs) and insects (26 MOTUs). Of the 1,314 MOTUs, 537 (40,9\%) were found in both lakes, 418 MOTUs were unique to location 1(De Ezelenwei), and 359 MOTUs unique to location 2

184 (De Drie Landjes).

The MOTU communities differed significantly between the two lakes for all twenty sampling moments, which is reflected in the NMDS plot based on the Sørenson dissimilarity matrix (Fig. 2). Clustering of samples into their respective lakes was supported by ANOSIM $(R=0.710, p=0.001)$. Similarly, ANOSIM also supported grouping of samples into two seasonal groups, spring (2 May to 13 June), and summer (20 June to 12 September) $(R=0.486, p=0.001)$. For the metazoan-only subset, the separation between 
191 the locations is still supported by ANOSIM, albeit not as clear as in the dataset with all MOTUs $(\mathrm{R}=$

$1920.424, p=0.001)$. The grouping into spring and summer is also supported $(R=0.587, p=0.001)$.

\section{PCR replicates}

195

196

197

Out of 1,314 MOTUs, 110 only ever occurred in one PCR replicate, with an average of $14.0 \pm 1.6$ (mean \pm SEM) reads. The other 1,204 MOTUs occurred on average in $21.2 \pm 1.1$ of the 356 total replicates. No MOTU was found in all replicates. Average Sørenson dissimilarity between PCR replicates was 0.26 (Fig. 3). Using the "additive" PCR processing strategy, samples had an average of $102.5 \pm 4.0$ MOTUs. Under the "relaxed" scenario samples had an average of $65.7 \pm 2.4$ MOTUs, and 280 MOTUs were discarded from the MOTU table. In the "strict" scenario an additional 246 MOTUs were discarded (Table 1). The remaining 788 MOTUs still represented $95.1 \%$ of the total read data. One replicate on average contained $70.9 \%$ of MOTUs found in the total spatial replicate sample (the three PCR replicates combined) (range 34.6-95.8\%), two replicates combined were able to detect an average of $88.4 \%$ of the MOTUs (range $55.8-100 \%$ ). In only ten of 120 samples, the addition of a third PCR replicate did not result in additional MOTUs found. Seven of the PCR replicates contained no MOTUs that could be identified as metazoan, two subsamples had no metazoan MOTUs in any of their PCR replicates. Average Sørenson dissimilarity between PCR replicates in the metazoan-only subset of the data was 0.18 (Fig. 3), although in some cases it was as high as 1.0.

\section{Sampling replicates}

Average Sørenson dissimilarity between sampling replicates within one location at the same time point was 0.48 using the "additive" PCR replicate strategy (Fig. 3), and significantly higher than dissimilarities between PCR replicates (t-test, $p=0.005)$. When using the "relaxed" and "strict" approaches, the average was slightly lower ( 0.45 and 0.46 , respectively) (Table 1 ), but not significantly different 
215 (ANOVA). Four samples with only two successful PCR replicates were omitted from this analysis. There

216 was a strong correlation between the Sørenson dissimilarity matrices for sample replicates under all

217 three PCR replicate processing strategies (Fig. S1), both for the dissimilarities between sampling

218 replicates pairs, and the dissimilarity matrix as a whole.

219

220 The high dissimilarity between sampling replicates was reflected in the contribution of each sampling

221 replicate to the total diversity of the lakes at each time point. The three sampling replicates combined

222 had an average of $187.3 \pm 9.1$ MOTUs, whereas a combination of two replicates only represented $81.0 \%$

223 (range 34.0-100\%) of that total. In only one of 40 (two lakes, 20 time points) cases, the addition of a

224 third sampling replicate did not provide additional MOTUs. One sampling replicate on average only

225 produced $103.0 \pm 3.9$ MOTUs, which represented 55.4\% of the total (range 12.1-92.5\%). Regardless of

226 the PCR replicate processing strategy used, the average proportion of MOTUs unique to one of three

227 sample replicates was roughly the same (Table 1 ).

228

229

Temporal replicates

230 To look at the temporal patterns in the data, we used the "additive" PCR processing strategy, and added

231 each of the three replicates per time per location into one data point. This resulted in 40 data points

232 with an average of $187.3 \pm 9.1$ MOTUs for $104,935 \pm 5,007$ reads. Again, there was no correlation

233 between number of reads and number of MOTUs. A total of 257 (19.6\%) MOTUs only ever occur in a

234 single time point in a single location, only four MOTUs occur every week in both locations. Weekly

235 samples represented between $9.5 \%$ and $37.9 \%$ (average $20.2 \%$ ) of the total MOTU community observed

236 in the lake, with later weeks generally having a higher richness than the earlier weeks. Turnover was not

237 calculated as it was inflated by MOTUs occurring in non-consecutive weeks.

238

PeerJ reviewing PDF | (2019:04:36312:1:1:NEW 15 May 2019) 
239 The average Sørenson dissimilarity between two replicates taken one week apart at the same sampling

240 point was 0.53 , which is significantly higher than the dissimilarity between two replicates taken at the

241 same time (t-test, $p=0.005$ ) (Fig. 3). With the sampling replicates combined, the Sørenson dissimilarity

242 between the total communities of one location a week apart was 0.48 on average. Looking at larger time

243 intervals, there was a significant correlation between interval duration and Sørenson dissimilarity

244 (Spearman correlation $\rho=0.812, p<0.001$ ) (Fig. 4).

For the metazoan-only subset, dissimilarity between the sampling replicates and the temporal replicates

was much higher than for the whole dataset, at 0.65 and 0.62 , but with no significant difference

intervals of three or more weeks (t-test, $p=0.002)$. The same effects as with all MOTUs were seen when

looking at the PCR replicate processing strategies, where average dissimilarities were not significantly

different for each of the three strategies, albeit much higher than when using all MOTUs (Table 1). The

correlation between interval duration and Sørenson dissimilarity was also significant for metazoan-only data (Spearman correlation $\rho=0.555, p<0.001$ ) (Fig. 4).

\section{DISCUSSION}

Our results demonstrate the relatively large differences that can exist between sampling replicates, both on a spatial and a temporal scale. A significant challenge in the use of eDNA for metabarcoding stems from the heterogeneity of eDNA within the environment, and also in DNA extracts. The latter introduces

260 a stochastic effect when sequencing multiple PCR replicates, in which less abundant species may not be

261 found in all replicates. We applied three ways of bioinformatics processing of PCR replicates: (1) using all

262 MOTUs ("additive"), (2) only using MOTUs present in two or more replicates ("relaxed"), and (3) only 
263

264

265

266

267

using MOTUs present in all three replicates ("strict") (Alberdi et al., 2018). Whilst the chosen strategy had an impact on the total and average number of MOTUs found in each sample, general patterns of dissimilarities between samples were not largely impacted.

When we look at the heterogeneity of eDNA across the three sampling replicates within one location at a given time, the proportion of MOTUs that occur in either one or in all of the samples stays the same regardless of PCR replicate processing strategy. This indicates that removal of MOTUs not covered by all PCR replicates (the "strict" strategy) does not necessarily make spatial replicates more similar. This observation is confirmed by the average dissimilarity between the spatial samples, which is not significantly different for any of the three PCR replicate strategies (Table 1). Similarly, the Sørenson dissimilarity matrices were highly correlated $(r=0.929$ and $r=0.917$ for "additive" versus "relaxed" and "relaxed" versus "strict", respectively. Pearson correlation, $p<0.001$ ) (Fig. S1). This suggests that the selected strategy can vary depending on the research question without significantly impacting observed patterns of biodiversity, although it affects the richness estimates. PCR results are not always reproducible, as witnessed by the average dissimilarity of 0.26 between PCR replicates in this study, but also as reported in the detection of rare species (e.g. Ficetola et al. 2008, Buxton et al. 2018). Especially when looking for rare species, multiple PCR replicates improve detection chances. For analyses that benefit from more complete taxa lists, such as those performed for WFD monitoring, the inclusion of multiple PCR replicates also seems beneficial. While we only took three sampling replicates within each lake in each week, others have suggested as much as nine samples to estimate biodiversity from eDNA (Grey et al., 2018).

\section{Compared to PCR replicates, the Sørenson dissimilarity between spatial replicates ( 0.48 on average for} the full dataset, 0.65 for the metazoan-only subset) is significantly higher (Fig. 3), which reflects the 
287 heterogeneity of eDNA within the environment. Previous studies have already pointed out that eDNA 288 signal can have strong local effects (Moyer et al., 2014; O’Donnell et al., 2017; Stewart et al., 2017), due

289 to limited dispersal and sedimentation, but also the rapid degradation of eDNA (Dejean et al., 2011;

290 Barnes \& Turner, 2015). The use of spatial replicate sampling to retrieve eDNA results that are

291 representative for the whole body of water has been stressed (Goldberg et al., 2016; Harper et al.,

292 2019), and shown to improve eDNA monitoring efficacy (Goldberg, Strickler \& Fremier, 2018).

293 Resampling at different time points, however, has received little attention. Up until now research into

294 seasonal variation has often focused on a limited set of temporal samples, such as spring versus

295 autumn/winter (Chain et al., 2016; Guardiola et al., 2016; Lacoursière-Roussel et al., 2018). The effects

296 of temporal replicate sampling in this study were comparable with those of spatial replicates, with

297 dissimilarities between samples taken at one sampling point a week apart slightly but significantly higher

298 than those between samples taken within one lake at a certain week (average 0.53 versus 0.48) (Fig. 3).

299 Almost a fifth (19.6\%) of MOTUs was only ever detected in one week. In the metazoan-only subset the

300 spatial and temporal dissimilarities were higher than for the complete dataset $(0.65$ and 0.62 ,

301 respectively), although not significantly different from each other. Temporal dissimilarity was

302 significantly higher than spatial dissimilarity, however, for intervals of three weeks or more. Similar

303 observations were made for example in fish (Stoeckle, Soboleva \& Charlop-Powers, 2017; Sigsgaard et

304 al., 2017), where many species were only detected in a few time points, showing that temporal sampling

305 regimes are needed for optimal recovery of the total biodiversity. Our sampling timeframe coincides

306 with the period in which most of the traditional WFD monitoring is performed, for which insights into

307 within-season community changes are more relevant than between-season variations.

309 The data included a number of MOTUs occurring in non-consecutive weeks, suggesting these MOTUs

310 went undetected, rather than being absent from the environment. A detection/non-detection cannot be 
311 directly translated into presence/absence (Roussel et al., 2015). These irregular patterns of occurrence

312 may have increased the dissimilarity between replicate samples, both temporal and spatial. However,

313 we observed a strong correlation between time interval and Sørenson dissimilarity (Spearman

314 correlation, $\rho=0.812 p<0.001$ ) (Fig. 4). Interestingly, it is not a linear correlation, and there seems to be

315 a maximum to the dissimilarity between samples taken at different time points. Although we only

316 sampled for 20 consecutive weeks, this data suggest that the community never changes completely

317 within this time frame. The maximum observed Sørenson dissimilarity between two samples taken at

318 one sampling point is 0.90 (for a nine week interval). This indicates that, even though there are large

319 changes in eDNA composition between different time points, there is some basal community that is

320 present throughout the sampling period and does not change. Such basal communities could be

321 relevant for identifying potential novel targets for eDNA-based monitoring, as it would allow for a time-

322 independent assessment. Planktonic crustaceans, such as the copepods and branchiopods found in

323 relatively large numbers (both MOTUs and reads, Figs S2-S3) have the potential to be such new bio-

324 indicators, as they may be more easily detected using eDNA and likely to respond quicker to

325 environmental changes (Lim et al., 2016; Montagud et al., 2018). Additionally, we observed a linear

326 increase in dissimilarity between the two locations over time (Pearson correlation, $r=0.551, p<0.001$ ).

327 Average Sørenson dissimilarity of the two lakes was 0.71 when sampled in the same week (interval=0),

328 and increased up to 0.80 when sampled 19 weeks apart (Fig. 4). This indicates that studies comparing

329 communities between locations should be wary of the time intervals between sampling, as larger

330 intervals between sampling may lead to inflated dissimilarities.

332 Even though there are large differences between communities along the temporal gradient, there were 333 no large shifts in the taxonomic compositions defined by LCA (Figs S2-S3). Other than an increase in the 334 number of metazoan taxa over time (both in absolute number of MOTUs and in proportion of the total 
diversity), the proportional contribution of each of the different taxonomic groups is roughly the same

336 for all twenty weeks, in both lakes. This indicates that seasonal succession mostly occurs within the

337 taxonomic groups. The increase in metazoan taxa may be slightly inflated in the data for location 2 ,

338 where algae (2 MOTUs classified as Chrysophyceae) dominated the reads between 30 May and 20 June,

339 and potently out competed others in both DNA extraction and amplification. The rest of the weeks in

340 location 2, and all weeks in location 1 were mostly dominated by arthropod (copepod and branchiopod)

341 and unidentified reads (average of $36.0 \%$ and $48.7 \%$, respectively).

342

343 The primers used in this study perform well on macroinvertebrate bulk samples, but are degenerate

enough to amplify a wide range of non-target DNA from non-metazoan sources present in

environmental samples that would normally not be found in bulk macroinvertebrate samples (Fig. 1). In

our case, only $13.4 \%$ of the MOTUs could be assigned to metazoan phyla. Within those, only about a

347 third (51 out of 176) could be assigned to phyla that are actually counted as macroinvertebrates for the

purpose of traditional quality monitoring under the WFD. The remainder of the metazoans were mainly

branchiopods and copepods. Similar results with non-target taxa were reported in other papers using

degenerate COI primers for freshwater community metabarcording (e.g. Weigand \& Macher, 2018).

There has been some debate about the usability of the standard COI barcode region defined by Hebert

et al. (2003) within DNA- and eDNA-based analyses, but thus far the benefit of an extensive COI

database seems to outweigh the drawbacks (Andújar et al., 2018), as also witnessed by the many primer 
359 can be identified up to species level, unidentified (or partially identified, in the form of higher taxa)

360 MOTUs can still be matched across different samples and may therefore still be of use for community

361 analyses (Lim et al., 2016).

362

363 The primer sets used in this study may not have been optimal for recovery of all taxon groups, and

364 group-specific primers may be more appropriate for the detection of novel bio-indicators. Nonetheless,

365 we expect the temporal effects observed in this study to play a role in any community analysis. Even

366 when eDNA is used for BQE monitoring, time intervals between sampling sites will likely remain, as it

367 practically impossible to sample and process all sites within a short timeframe. Seasonal effects have

368 been reported in the rich history of publications based on morphological observation of seasonality in

369 planktonic organisms (e.g. Gosselain et al. 1994, Wu et al. 2013), but molecular tools will allow for much

370 finer resolution observations. We strongly encourage any research into the use of novel indicator taxa to

371 take these temporal changes into consideration, as they clearly affect non-macroinvertebrate taxa such

372 as the phyto- and zooplankton groups observed in this study.

373

374 CONCLUSIONS

375

376 We here present the first study that directly compares the effects of small-scale spatial and temporal

377 resampling eDNA for metabarcoding. We show that replication leads to better estimations of total

378 biodiversity, where the effects of spatiotemporal sampling replicates are significantly greater than PCR

379 replications, even though the latter can already bring a substantial increase in richness depending on the

380 replicate processing strategy. Interestingly, the PCR replicate handling strategy has little effect on

381 patterns in biodiversity and dissimilarity between samples, and there are no severe drawbacks of

382 including even those MOTUs that occur in only one replicate. Dissimilarities between temporally

Peer] reviewing PDF | (2019:04:36312:1:1:NEW 15 May 2019) 
383

384 385

separated samples were approximately equivalent to the dissimilarities between spatially separated samples. These dissimilarities increase over longer time intervals, suggesting that population turnover effects are stronger than community heterogeneity. This is an important consideration for any study comparing multiple communities that have been sampled at different time points, as well as any study that delves into the use of novel bio-indicators. Non-macroinvertebrate taxa, such as the phyto- and zooplankton groups observed in this study, are often put forward as potential bio-indicators. The effects of sampling strategies, especially short-term temporal replicate sampling, can have a considerate impact on the usability of these taxa.

\section{ACKNOWLEDGEMENTS}

We thank Staatsbosbeheer and Casper Zuyderduyn for access to the dune lakes and permits for field work. We also thank Dr. Oscar Vorst for his help in assessing potential sampling sites, Jeroen Visser for his assistance in the sample collection and laboratory work, and Elza Duijm for her assistance with NGS library preparations.

\section{REFERENCES}

Afgan E., Baker D., Batut B., Van Den Beek M., Bouvier D., Ech M., Chilton J., Clements D., Coraor N., Grüning BA., Guerler A., Hillman-Jackson J., Hiltemann S., Jalili V., Rasche H., Soranzo N., Goecks J., Taylor J., Nekrutenko A., Blankenberg D. 2018. The Galaxy platform for accessible, reproducible and collaborative biomedical analyses: 2018 update. Nucleic Acids Research 46:W537-W544. DOI: 10.1093/nar/gky379.

Alberdi A., Aizpurua O., Gilbert MTP., Bohmann K. 2018. Scrutinizing key steps for reliable 
407

408

409

410

411

412

413

414

415

416

417

418

419

420

421

422

423

424

425

426

427

428

429

430

metabarcoding of environmental samples. Methods in Ecology and Evolution 9:134-147. DOI: 10.1111/2041-210X.12849.

Andújar C., Arribas P., Yu DW., Vogler AP., Emerson BC. 2018. Why the COI barcode should be the community DNA metabarcode for the Metazoa. Molecular Ecology 27:3968-3975. DOI: 10.1111/mec.14844.

Barnes MA., Turner CR. 2015. The ecology of environmental DNA and implications for conservation genetics. Conservation Genetics 17:1-17. DOI: 10.1007/s10592-015-0775-4.

Barsoum N., Bruce C., Forster J., Ji Y-Q., Yu DW. 2019. The devil is in the detail: Metabarcoding of arthropods provides a sensitive measure of biodiversity response to forest stand composition compared with surrogate measures of biodiversity. Ecological Indicators 101:313-323. DOI: 10.1016/J.ECOLIND.2019.01.023.

Baselga A., Fujisawa T., Crampton-Platt A., Bergsten J., Foster PG., Monaghan MT., Vogler AP. 2013. Whole-community DNA barcoding reveals a spatio-temporal continuum of biodiversity at species and genetic levels. Nature Communications 4:1892. DOI: 10.1038/ncomms2881.

Benson DA., Karsch-Mizrachi I., Lipman DJ., Ostell J., Wheeler DL. 2005. GenBank. Nucleic Acids Research 33:D34-8. DOI: 10.1093/nar/gki063.

Bista I., Carvalho GR., Walsh K., Seymour M., Hajibabaei M., Lallias D., Christmas M., Creer S. 2017. Annual time-series analysis of aqueous eDNA reveals ecologically relevant dynamics of lake ecosystem biodiversity. Nature Communications 8:14087. DOI: 10.1038/ncomms14087.

Buxton AS., Groombridge JJ., Griffiths RA. 2018. Seasonal variation in environmental DNA detection in sediment and water samples. PLOS ONE 13:e0191737. DOI: 10.1371/journal.pone.0191737.

Camacho C., Coulouris G., Avagyan V., Ma N., Papadopoulos J., Bealer K., Madden TL. 2009. BLAST+: architecture and applications. BMC Bioinformatics 10:421. DOI: 10.1186/1471-2105-10-421.

Chain FJJ., Brown EA., Maclsaac HJ., Cristescu ME. 2016. Metabarcoding reveals strong spatial structure 
431

432

433

434

435

436

437

438

439

440

441

442

443

444

445

446

447

448

449

450

451

452

453

454

and temporal turnover of zooplankton communities among marine and freshwater ports. Diversity and Distributions 22:493-504. DOI: 10.1111/ddi.12427.

Clarke RT., Hering D. 2006. Errors and uncertainty in bioassessment methods - major results and conclusions from the STAR project and their application using STARBUGS. Hydrobiologia 566:433439. DOI: 10.1007/s10750-006-0079-2.

Dejean T., Valentini A., Duparc A., Pellier-Cuit S., Pompanon F., Taberlet P., Miaud C. 2011. Persistence of environmental DNA in freshwater ecosystems. PLOS ONE 6:e23398. DOI: 10.1371/journal.pone.0023398.

Dejean T., Valentini A., Miquel C., Taberlet P., Bellemain E., Miaud C. 2012. Improved detection of an alien invasive species through environmental DNA barcoding: the example of the American Bullfrog Lithobates catesbeianus. Journal of Applied Ecology 49:953-959. DOI: 10.1111/j.13652664.2012.02171.x.

Edgar RC. 2016. UNOISE2: improved error-correction for Illumina 165 and ITS amplicon sequencing. Cold Spring Harbor Laboratory. DOI: 10.1101/081257.

Elbrecht V., Leese F. 2017. Validation and Development of COI Metabarcoding Primers for Freshwater Macroinvertebrate Bioassessment. Frontiers in Environmental Science 5:11. DOI: 10.3389/fenvs.2017.00011.

Ficetola GF., Miaud C., Pompanon F., Taberlet P. 2008. Species detection using environmental DNA from water samples. Biology Letters 4:423-5. DOI: 10.1098/rsbl.2008.0118.

Goldberg CS., Strickler KM., Fremier AK. 2018. Degradation and dispersion limit environmental DNA detection of rare amphibians in wetlands: Increasing efficacy of sampling designs. Science of The Total Environment 633:695-703. DOI: 10.1016/J.SCITOTENV.2018.02.295.

Goldberg CS., Turner CR., Deiner K., Klymus KE., Thomsen PF., Murphy MA., Spear SF., McKee A., OylerMcCance SJ., Cornman RS., Laramie MB., Mahon AR., Lance RF., Pilliod DS., Strickler KM., Waits LP., 
455

456

457

458

459

460

461

462

463

464

465

466

467

468

469

470

471

472

473

474

475

476

477

478

Fremier AK., Takahara T., Herder JE., Taberlet P. 2016. Critical considerations for the application of environmental DNA methods to detect aquatic species. Methods in Ecology and Evolution. DOI: 10.1111/2041-210X.12595.

Gosselain V., Descy JP., Everbecq E. 1994. The phytoplankton community of the River Meuse, Belgium: seasonal dynamics (year 1992) and the possible incidence of zooplankton grazing. Hydrobiologia 289:179-191. DOI: 10.1007/BF00007419.

Grey EK., Bernatchez L., Cassey P., Deiner K., Deveney M., Howland KL., Lacoursière-Roussel A., Leong SCY., Li Y., Olds B., Pfrender ME., Prowse TAA., Renshaw MA., Lodge DM. 2018. Effects of sampling effort on biodiversity patterns estimated from environmental DNA metabarcoding surveys. Scientific Reports 8:8843. DOI: 10.1038/s41598-018-27048-2.

Guardiola M., Wangensteen OS., Taberlet P., Coissac E., Uriz MJ., Turon X. 2016. Spatio-temporal monitoring of deep-sea communities using metabarcoding of sediment DNA and RNA. PeerJ 4:e2807. DOI: 10.7717/peerj.2807.

Hänfling B., Handley LL., Read DS., Hahn C., Li J., Nichols P., Blackman RC., Oliver A., Winfield IJ. 2016. Environmental DNA metabarcoding of lake fish communities reflects long-term data from established survey methods. Molecular Ecology 25:3101-3119. DOI: 10.1111/mec.13660.

Harper LR., Buxton AS., Rees HC., Bruce K., Brys R., Halfmaerten D., Read DS., Watson H V., Sayer CD., Jones EP., Priestley V., Mächler E., Múrria C., Garcés-Pastor S., Medupin C., Burgess K., Benson G., Boonham N., Griffiths RA., Lawson Handley L., Hänfling B. 2019. Prospects and challenges of environmental DNA (eDNA) monitoring in freshwater ponds. Hydrobiologia 826:25-41. DOI: 10.1007/s10750-018-3750-5.

Hebert PDN., Cywinska A., Ball SL., deWaard JR. 2003. Biological identifications through DNA barcodes. Proceedings of the Royal Society B: Biological Sciences 270:313-21. DOI: 10.1098/rspb.2002.2218.

Huson DH., Auch AF., Qi J., Schuster SC. 2007. MEGAN analysis of metagenomic data. Genome research 

17:377-86. DOI: 10.1101/gr.5969107.

480 481

484

485

486

487

488

489

490

491

492

493

494

495

496

497

498

499

500

501

502

Joshi NA., Fass JN. 2011. Sickle: A sliding-window, adaptive, quality-based trimming tool for FastQ files (Version 1.33). Available at https://github.com/najoshi/sickle.

Klymus KE., Marshall NT., Stepien CA. 2017. Environmental DNA (eDNA) metabarcoding assays to detect invasive invertebrate species in the Great Lakes. PLOS ONE 12:e0177643. DOI: 10.1371/journal.pone.0177643.

Lacoursière-Roussel A., Howland K., Normandeau E., Grey EK., Archambault P., Deiner K., Lodge DM., Hernandez C., Leduc N., Bernatchez L. 2018. eDNA metabarcoding as a new surveillance approach for coastal Arctic biodiversity. Ecology and Evolution 8:7763-7777. DOI: 10.1002/ece3.4213.

Leray M., Yang JY., Meyer CP., Mills SC., Agudelo N., Ranwez V., Boehm JT., Machida RJ. 2013. A new versatile primer set targeting a short fragment of the mitochondrial COI region for metabarcoding metazoan diversity: application for characterizing coral reef fish gut contents. Frontiers in Zoology 10:34. DOI: 10.1186/1742-9994-10-34.

Lim NKM., Tay YC., Srivathsan A., Tan JWT., Kwik JTB., Baloğlu B., Meier R., Yeo DCJ. 2016. Nextgeneration freshwater bioassessment: eDNA metabarcoding with a conserved metazoan primer reveals species-rich and reservoir-specific communities. Royal Society Open Science 3:160635. DOI: 10.1098/rsos.160635.

Magoč T., Salzberg SL. 2011. FLASH: Fast length adjustment of short reads to improve genome assemblies. Bioinformatics 27:2957-2963. DOI: 10.1093/bioinformatics/btr507.

Martin M. 2011. Cutadapt removes adapter sequences from high-throughput sequencing reads. EMBnet.journal 17:10. DOI: 10.14806/ej.17.1.200.

Montagud D., Soria JM., Soria-Perpiñà X., Alfonso T., Vincente E. 2018. A comparative study of four indexes based on zooplankton as trophic state indicators in reservoirs. Limnetica 38. DOI: 10.23818/limn.38.06 
503 Moyer GR., Díaz-Ferguson E., Hill JE., Shea C. 2014. Assessing environmental DNA detection in controlled $504 \quad$ lentic systems. PLOS ONE 9:e103767. DOI: 10.1371/journal.pone.0103767.

505 O’Donnell JL., Kelly RP., Shelton AO., Samhouri JF., Lowell NC., Williams GD. 2017. Spatial distribution of 506 environmental DNA in a nearshore marine habitat. PeerJ 5:e3044. DOI: 10.7717/peerj.3044.

507 Oksanen J., Kindt R., Legendre P., O’Hara R. 2007. Vegan: Community Ecology Package. R package 508 version 2.4-6. https://www.rdocumentation.org/packages/vegan.

509 Olds BP., Jerde CL., Renshaw MA., Li Y., Evans NT., Turner CR., Deiner K., Mahon AR., Brueseke MA., 510 Shirey PD., Pfrender ME., Lodge DM., Lamberti GA. 2016. Estimating species richness using 511 environmental DNA. Ecology and Evolution. DOI: 10.1002/ece3.2186.

512 Rees HC., Baker CA., Gardner DS., Maddison BC., Gough KC. 2017. The detection of great crested newts 513 year round via environmental DNA analysis. BMC Research Notes 10:327. D0I: 10.1186/s13104$514 \quad$ 017-2657-y.

515 Rognes T., Flouri T., Nichols B., Quince C., Mahé F. 2016. VSEARCH: a versatile open source tool for 516 metagenomics. PeerJ 4:e2584. DOI: 10.7717/peerj.2584.

517 Roussel J-M., Paillisson J-M., Tréguier A., Petit E. 2015. The downside of eDNA as a survey tool in water 518 bodies. Journal of Applied Ecology 52:n/a-n/a. DOI: 10.1111/1365-2664.12428.

Schmidt BR., Kéry M., Ursenbacher S., Hyman OJ., Collins JP. 2013. Site occupancy models in the analysis of environmental DNA presence/absence surveys: A case study of an emerging amphibian

521 pathogen. Methods in Ecology and Evolution 4. DOI: 10.1111/2041-210X.12052.

522

Schmieder R., Edwards R. 2011. Quality control and preprocessing of metagenomic datasets. Bioinformatics 27:863-864. DOI: 10.1093/bioinformatics/btr026.

524 Schneider J., Valentini A., Dejean T., Montarsi F., Taberlet P., Glaizot O., Fumagalli L. 2016. Detection of 525 invasive mosquito vectors using environmental DNA (eDNA) from water samples. PLoS ONE 526 11:e0162493. DOI: 10.1371/journal.pone.0162493. 
527 Sigsgaard EE., Nielsen IB., Carl H., Krag MA., Knudsen SW., Xing Y., Holm-Hansen TH., Møller PR.,

528 Thomsen PF. 2017. Seawater environmental DNA reflects seasonality of a coastal fish community.

529 Marine Biology 164:128. DOI: 10.1007/s00227-017-3147-4.

530 Stewart K., Ma H., Zheng J., Zhao J. 2017. Using environmental DNA to assess population-wide

531 spatiotemporal reserve use. Conservation Biology 31:1173-1182. DOI: 10.1111/cobi.12910.

532 Stoeckle MY., Soboleva L., Charlop-Powers Z. 2017. Aquatic environmental DNA detects seasonal fish

533 abundance and habitat preference in an urban estuary. PLOS ONE 12:e0175186. DOI:

$534 \quad$ 10.1371/journal.pone.0175186.

535 Thomsen PF., Kielgast J., Iversen LL., Wiuf C., Rasmussen M., Gilbert MTP., Orlando L., Willerslev E. 2012.

536 Monitoring endangered freshwater biodiversity using environmental DNA. Molecular Ecology

537 21:2565-73. DOI: 10.1111/j.1365-294X.2011.05418.x.

538 Turner CR., Miller DJ., Coyne KJ., Corush J. 2014. Improved methods for capture, extraction, and

539 quantitative assay of environmental DNA from Asian bigheaded carp (Hypophthalmichthys spp.).

$540 \quad$ PLOS ONE 9:e114329. DOI: 10.1371/journal.pone.0114329.

541 Weigand AM., Macher J-N. 2018. A DNA metabarcoding protocol for hyporheic freshwater meiofauna:

$542 \quad$ Evaluating highly degenerate $\mathrm{COI}$ primers and replication strategy. Metabarcoding and

$543 \quad$ Metagenomics 2:e26869. DOI: 10.3897/mbmg.2.26869.

544 Wu Z., Cai Y., Liu X., Xu CP., Chen Y., Zhang L. 2013. Temporal and spatial variability of phytoplankton in

545 Lake Poyang: The largest freshwater lake in China. Journal of Great Lakes Research 39:476-483.

$546 \quad$ DOI: 10.1016/j.jgIr.2013.06.008.

547 


\section{Figure 1}

Taxonomic assignment of MOTUs.

Taxonomic assignments of the MOTUs at (A) phylum-level and (B) class-level for metazoa, using a lowest common ancestor approach in MEGAN. Numbers in the pie chart indicate the number of MOTUs assigned to each phylum. 
A

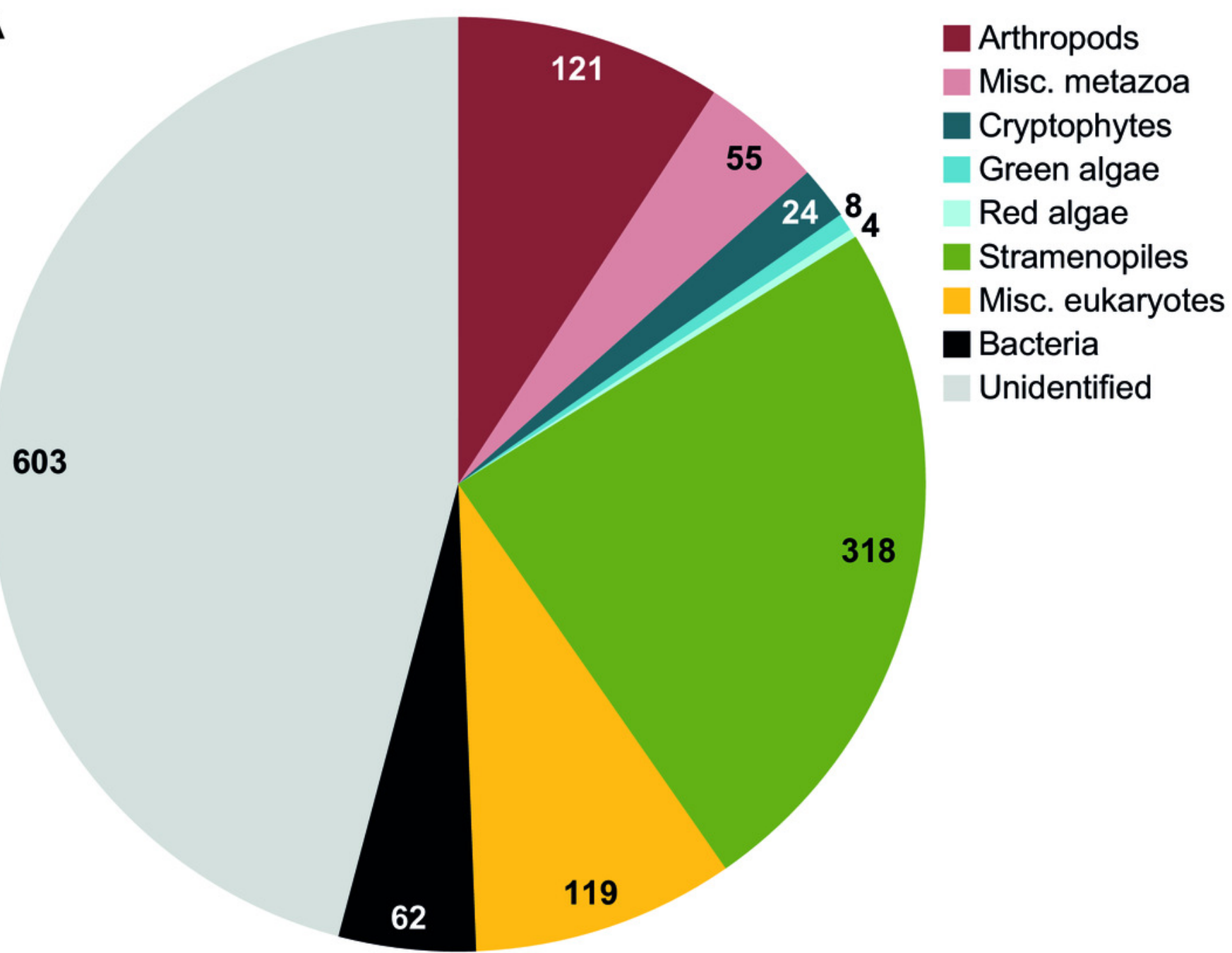

\section{B Metazoa}

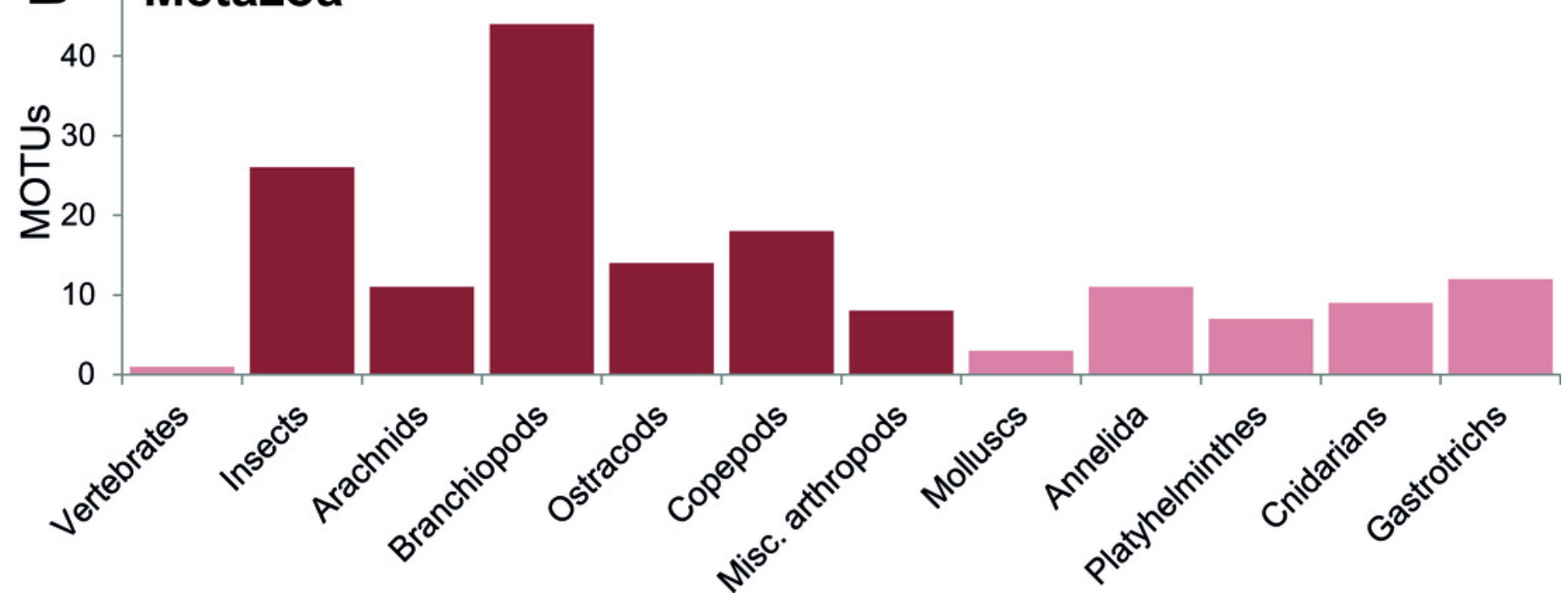




\section{Figure 2}

NMDS plots representing the dissimilarities between sampling sites.

Two-dimensional NMDS plots constructed based on Sørenson dissimilarities between sampling sites using (A) all MOTUs and (B) only metazoan MOTUs. 2D stress values are displayed in the panels. Each point represents the combined community of the three spatial sampling replicates taken at each of the two locations on each of the 20 time points, with the PCR replicates combined using the "additive" strategy. Shapes indicate the location, colors are used to indicate the month in which samples were obtained, with numbers labeling the consecutive weeks from 2 May to 12 September. ANOSIM supported grouping of the samples belonging to one lake for both all MOTUs as the metazoan-only dataset $(R=0.710$ and $R=$ 0.424 , respectively, $p=0.001$ ). Seasonal grouping was similarly supported by ANOSIM, splitting samples into two seasonal groups (2 May to 13 June, and 20 June to 12 September) $(R=0.486$ and $R=0.587$, respectively, $p=0.001)$ for all MOTUs and metazoan-only.
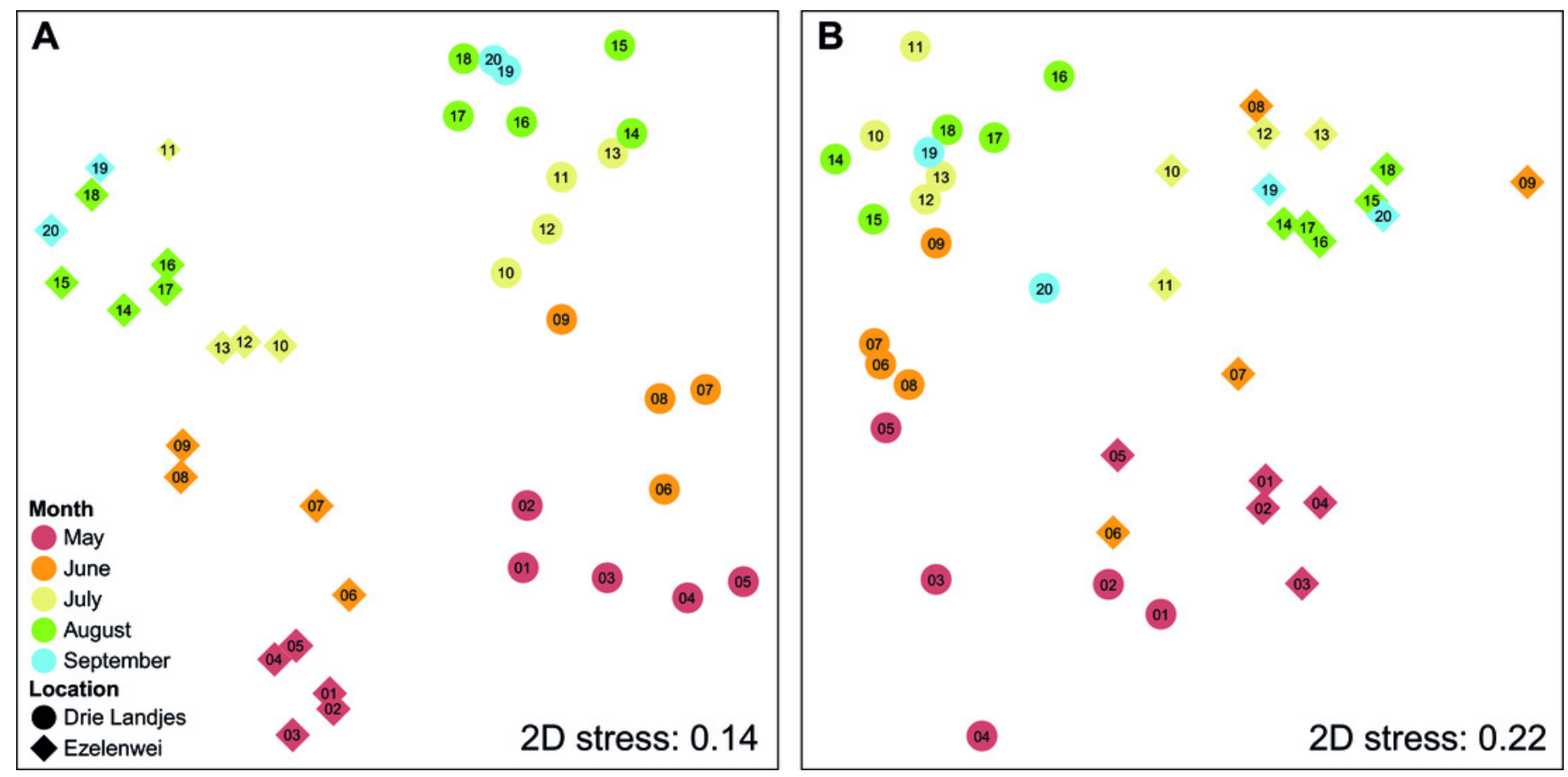


\section{Figure 3}

Average dissimilarities between PCR replicates, spatial replicates, and temporal replicates.

(A) Schematic representation of the replicate sampling strategies and (B) boxplot displaying Sørenson dissimilarity values for PCR replicates (green, $n=352$ for all MOTUs, $n=344$ for metazoa), spatial sampling replicates (red, $n=104$ ) and temporal replicates separated by one week (blue, $n=100$ ) for both all MOTUs and metazoan-only MOTUs. In both cases, the dissimilarity between spatial replicates was significantly higher than between PCR replicates (t-test, $p=0.005$ ). Only in the case of all MOTUs was the temporal dissimilarity significantly higher than the spatial dissimilarity (t-test, $p=0.005$ ). There was no significant difference between spatial and temporal dissimilarities in the metazoan-only for samples taken one week apart.
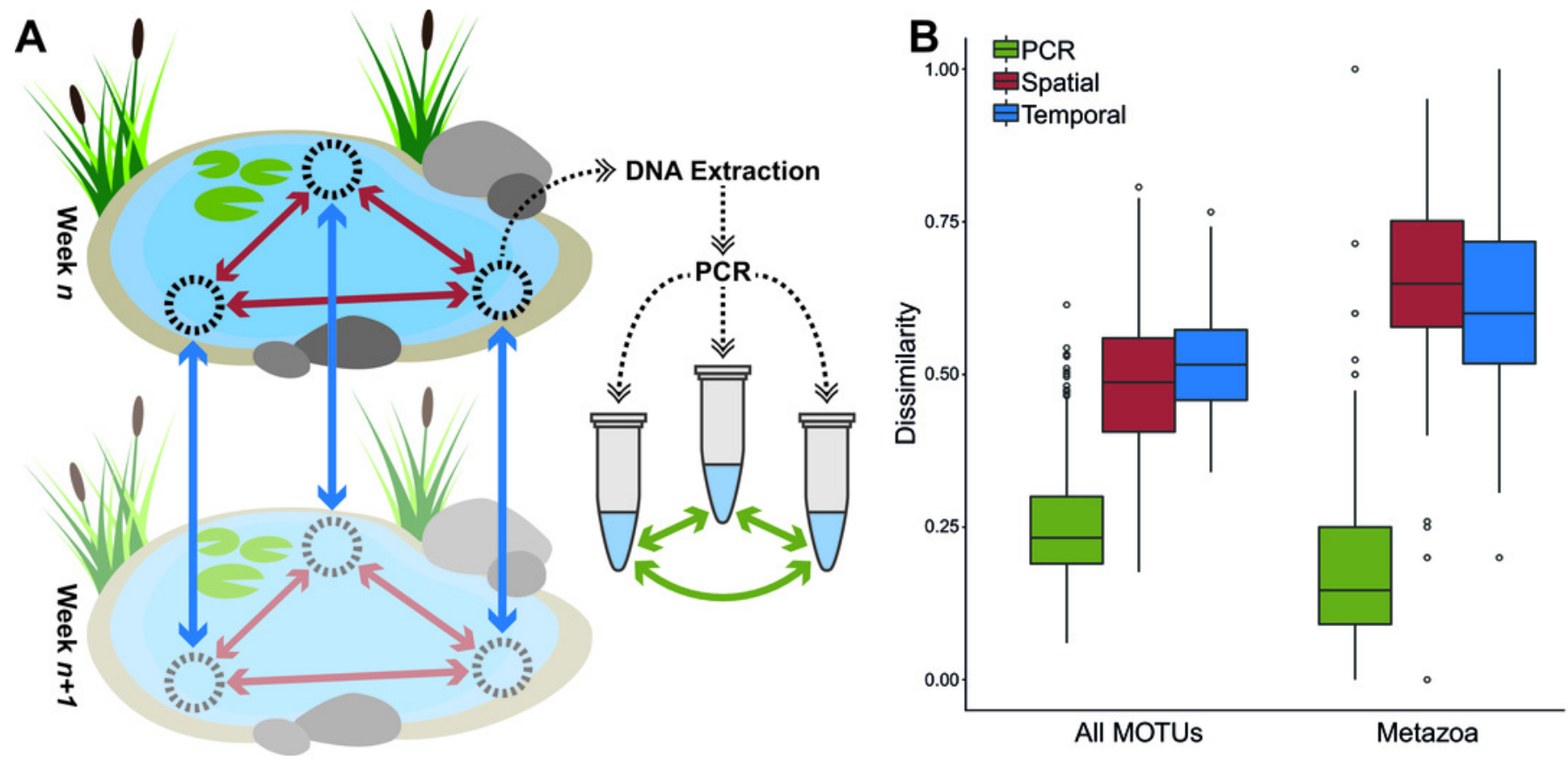


\section{Figure 4}

Correlations between time interval and sample dissimilarity.

Time interval between two sampling moments versus the Sørenson dissimilarity between

total communities for samples taken in the same lake, with (A) all MOTUs and (B) only

metazoan MOTUs (Spearman correlation, $p<0.001$ ), and time interval between two

sampling moments versus the Sørenson dissimilarity between total communities for samples

taken in different lakes, with (C) all MOTUs and (D) only metazoan MOTUs (Pearson

correlation, $p<0.001$ ) (with 95\% confidence interval). Correlation values are provided in the panels. Sampling replicates are merged into one sample per location per week, PCR replicates are processed using the "additive" strategy. 

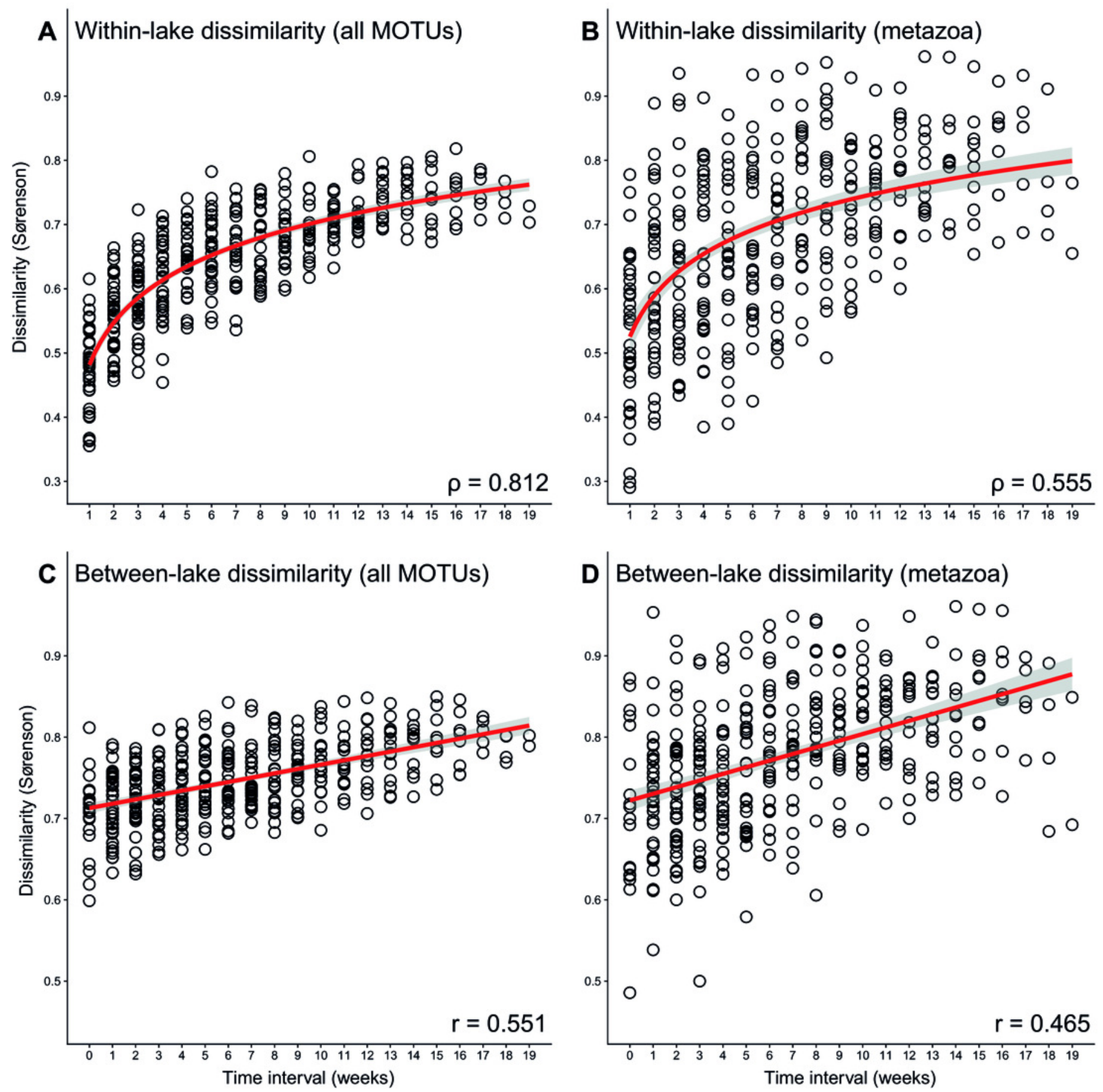


\section{Table $\mathbf{1}$ (on next page)}

Sample richness and heterogeneity under different replicate processing strategies.

Total richness of all samples combined, as well as average (mean \pm SEM) richness for each of the two locations at each of the 20 time points under different PCR replicate processing strategies ("additive", "relaxed", and "strict"), the effects on heterogeneity of MOTUs in the sample replicates and the average Sørenson dissimilarities between the sampling replicates (mean \pm SEM). For each of the three strategies, the MOTUs are divided into three categories:

(1) those MOTUs that are common and appear in all three sampling replicates; (2) MOTUs that are shared, and occur in two of three replicates; and (3) unique MOTUs, that only occur in a single sample replicate. 


\begin{tabular}{|l|l|l|l|l|l|l|l|}
\hline \multirow{2}{*}{ MOTUs } & \multirow{2}{*}{ PCR strategy } & \multicolumn{2}{l|}{ Richness } & \multicolumn{2}{l|}{ Sample Replicates } \\
\cline { 3 - 8 } & & \multicolumn{2}{l}{ Total } & Average & \multicolumn{2}{l|}{ Common (3/3) } & \multicolumn{2}{l|}{ Shared (2/3) } & \multicolumn{2}{l|}{ Unique (1/3) } & Dissimilarity \\
\hline \multirow{3}{*}{ All } & Additive (1/3) & 1314 & $187.3 \pm 9.1$ & $41.6(22.2 \%)$ & $37.5(20.0 \%)$ & $108.2(57.8 \%)$ & $0.48 \pm 0.01$ \\
& Relaxed (2/3) & 1034 & $114.8 \pm 5.8$ & $29.3(25.5 \%)$ & $23.8(20.7 \%)$ & $61.5(53.8 \%)$ & $0.46 \pm 0.01$ \\
& Strict (3/3) & 788 & $81.8 \pm 4.4$ & $17.6(21.5 \%)$ & $18.8(22.9 \%)$ & $45.5(55.6 \%)$ & $0.46 \pm 0.01$ \\
\hline \multirow{3}{*}{ Metazoan } & Additive (1/3) & 176 & $25.0 \pm 2.4$ & $18.0(75.2 \%)$ & $4.8(17.5 \%)$ & $2.3(10.9 \%)$ & $0.65 \pm 0.01$ \\
& Relaxed (2/3) & 156 & $19.2 \pm 2.3$ & $14.2(72.2 \%)$ & $3.8(17.7 \%)$ & $1.7(10.1 \%)$ & $0.66 \pm 0.02$ \\
& Strict (3/3) & 141 & $15.8 \pm 2.2$ & $12.0(75.2 \%)$ & $2.6(17.2 \%)$ & $1.1(7.6 \%)$ & $0.68 \pm 0.02$ \\
\hline
\end{tabular}

1 